\title{
Classification of Skin Melanoma using ANN
}

\author{
Jayant Ghode \\ Dept. of Electronic and Communication \\ S A T I Vidisha (MP), INDIA
}

\author{
Ashutosh Datar, PhD \\ (HOD) Dept. of Biomedical Engineering \\ S A T I Vidisha (MP), INDIA
}

\begin{abstract}
Cancer is one of the most deadly types of disease in the present era and skin cancer is one of them, an early detection of skin cancer can save many lives. Skin cancer occurs on the melanocytic cells of skin, so skin cancer is also known as malignant melanoma. It causes abnormal growth of melanocytic cells which produces sun protective pigment melanin. Due to melanin, melanoma appears as black or brown colour. For the detection of melanoma, conventional method is Biopsy. It is done by removing the skin sample and sample goes through a series of laboratory test. It is a time consuming process. It is more advantageous if computer based melanoma detection is used. This computer based detection contains imaging and artificial intelligence technique. In this paper we present novel approach for the detection of melanoma. This detection can be done with different steps- Dermatoscopy, Processing of image, Segmentation of region of interest, Feature extraction using Gray Level Co-occurrence Matrix (GLCM). These features are used for classification of cancerous and noncancerous melanoma using Back-Propagation Artificial Neural Network (ANN)
\end{abstract}

\section{General Terms}

Segmentation, Pattern Recognition, ROI, Classification

\section{Keywords}

Melanoma, Dermatoscopy, Segmentation, Gray Level Co-occurrence Matrix, Artificial Neural Network

\section{INTRODUCTION}

Skin is the largest organ in the human body. It is the outermost layer of human body and plays a major role in regulating body metabolisms and protecting against germs and other foreign bodies. There are many diseases that affect skin and the most dangerous of it is skin cancer. Human Body is made up of different types of cells. New cells replace older cells. Cancer is a condition when the cells growths are abnormal and in an uncontrolled way. In case of skin cancer, there is abnormal growth of melanocytic cells in the skin. Skin cancer appears as malignant or benign [1] [2]. Benign Melanoma is common and it is simply appearance of moles or tumours on the skin. Malignant Melanoma is appearance of sores that cause bleeding and it is the deadliest form of skin cancer. There are some specific features that distinguish Malignant Melanoma from the other three type of Benign Melanoma.

\subsection{Malignant Melanoma}

Malignant melanoma fig. 1 is named after the cell on which it occurs that is melanocytes. It produces pigment called melanin which protect from sun. Melanoma cell continues produce melanin, which result in cancers appearing in mixed shades of brown and black. There are four "ABCD" features by which early detection of malignant melanoma is done.

\subsubsection{Asymmetry}

Half part of the tumour does not match to the other half.

\subsubsection{Border Irregularity}

The border edges are ragged notched, blurred.

\subsubsection{Color}

Pigmentation with shades of tan, brown and black are present. It is not uniform.

\subsubsection{Diameter}

Diameter is greater than $6 \mathrm{~mm}$ and growing.
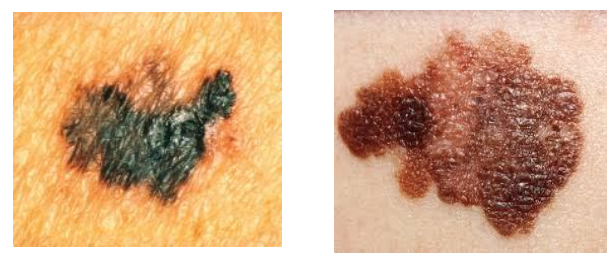

Fig.1 Malignant Melanoma

\subsection{Benign Melanoma}

The National Cancer Institute [4] described three type of benign melanoma which majorly occurs is Dysplastic Nevi (Moles) fig. 2 (a), Intradermal Nevi (Benign tumours) fig. 2 (b) and Seborrheic Keratoses (Benign tumours) fig. 2 (c). All three comes in the same category but differ in feature like age group of patient, color, shape, surface and size [3]. It is described in Table 1.

Table 1 Classification of Benign Melanoma

\begin{tabular}{|c|c|c|c|}
\hline Feature & $\begin{array}{l}\text { Dysplastic } \\
\text { Nevi }\end{array}$ & $\begin{array}{l}\text { Intra- } \\
\text { dermal } \\
\text { Nevi }\end{array}$ & $\begin{array}{l}\text { Seborrheic } \\
\text { Keratoses }\end{array}$ \\
\hline Patient & $\begin{array}{l}\text { Melanoma } \\
\text { cases in } \\
\text { family }\end{array}$ & $\begin{array}{l}\text { Children } \\
\text { and young }\end{array}$ & Older person \\
\hline Color & $\begin{array}{l}\text { Mix of tan, } \\
\text { brown, black } \\
\text { red }\end{array}$ & $\begin{array}{l}\text { Flesh- } \\
\text { colored, } \\
\text { pink or tan } \\
\text { and brown }\end{array}$ & $\begin{array}{c}\text { Tan and } \\
\text { brown or } \\
\text { fleshy or pink }\end{array}$ \\
\hline Shape & $\begin{array}{c}\text { Irregular } \\
\text { borders }\end{array}$ & $\begin{array}{c}\text { Round or } \\
\text { oval }\end{array}$ & $\begin{array}{l}\text { Oval and } \\
\text { round or } \\
\text { irregular }\end{array}$ \\
\hline Surface & $\begin{array}{l}\text { Smooth, } \\
\text { slightly scaly } \\
\text { or rough }\end{array}$ & $\begin{array}{l}\text { Smooth or } \\
\text { raised }\end{array}$ & $\begin{array}{l}\text { Rough, } \\
\text { veracious }\end{array}$ \\
\hline Size & $\begin{array}{l}>5 \mathrm{~mm} \\
\text { sometimes } \\
>10 \mathrm{~mm}\end{array}$ & $\begin{array}{l}<6 \mathrm{~mm} \text { in } \\
\text { diameter }\end{array}$ & $5-15 \mathrm{~mm}$ \\
\hline & & & \\
\hline
\end{tabular}




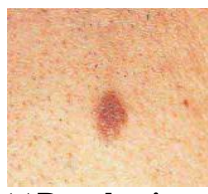

(a)Dysplastic

Nevi (b)Int

Nevi

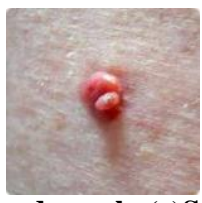

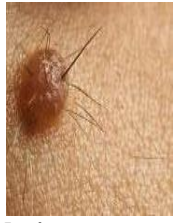

Keratoses
Fig. 2 Benign Melanoma

This description of malignant melanoma and benign melanoma indicate that malignant differs slightly in physical characteristics and colors. These features are used to distinguish malignant melanoma from other benign melanoma (benign tumour).Considering all the facts above, we proposed a computer based diagnosis of melanoma by which we can detect features of dermoscopic image and also classify it's type whether the given image is cancerous or noncancerous. This method uses both the image processing for feature extraction and artificial neural network for classification purpose.

\section{SKIN MELANOMA DETECTION SYSTEM}

Automatic skin cancer (melanoma) detection system is a classification system which classifies malignant melanoma and benign melanoma using imaging technique and a software MATLAB. This method uses Digital Image Processing (DIP) and Artificial Neural Network (ANN). Input to the system is color dermoscopic image, these images goes under the image processing step for removing the noise component. Dermoscopic images have both cancerous and healthy skin; our region of interest (ROI) is cancerous; so this cancerous region separated by segmenting it forms the healthy skin. For this purpose, segmentation technique is used. The unique features of malignant melanoma are extracting in the Feature Extraction Technique. Technique used here for feature extraction is Gray Level Co-occurrence Matrix (GLCM) [1] [12]. Feature obtain by GLCM are Contrast, Homogeneity, Correlation and Energy, along with these four feature, three additional features are Mean, Skewness and Kurtosis. Classification is done by Artificial Neural Network with these seven input neuron, hidden layers and one output neuron. The output of the ANN is ' 0 ' or ' 1 ', where 0 represents noncancerous case and ' 1 ' represents cancerous case.

\subsection{Dermatoscopy}

Dermatoscopy has invented new and fascinating morphological dimension of pigmented skin lesions. It also known as Dermoscopy or Epiluminescence Light Microscopy (ELM), It is a type of imaging technique used to examine skin lesions. In this process lens of microscope is placed directly on the skin lesion, Lighting can improve most of the pigmented color shades, structure; and allows direct analysis of lesion. Dermatoscopy with Digital Image Capture system fig. 3 (a) used for capture of Dermoscopic image fig.3 (b).

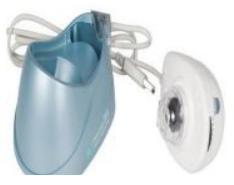

(a) Digital Image

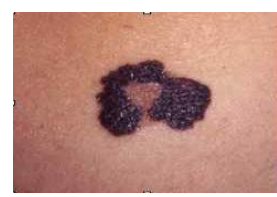

(b) Dermoscopic

\section{Capture System}

Image

Fig. 3 Dermatoscopy

\subsection{Image processing}

Digitally capture dermoscopic image undergo various Digital Image Processing Technique. The images consist of hairs and other noise component; and these cause inaccuracies in the detection of melanoma. To avoid that, these dermoscopic images are proceeding through various image processing technique. Image processing divided in to two parts Pre-processing and Postprocessing [2]. Image Pre-processing [1] [5] is the removal of noises in the image, like Hairs are removed using software 'Dull Razor' fig.4. 'Dull Razor' [10] is software used in dermatological cases. Post-processing is done for enhancing shape and edges of image, like contrast enhancement, sharpens the image border that improves accuracy of image for further steps.

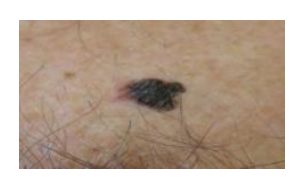

(a) Image with hair

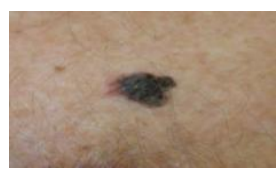

(b) Image after hair

Removal
Fig. 4 Hair removal using Dull Razor

\subsection{Segmentation}

In Image analysis Segmentation plays a major role. Segmentation subdivides an image into its continuous regions or object. After pre-processing and postprocessing image contain cancerous region and skin. The Region of interest (ROI) is cancerous region; and it has to be extracted from the healthy skin. The segmentation technique used is Active contour [6] [7] [13]; it segments the image in to foreground (ROI) and background (skin) fig.5 (b).In this technique is specified an initial guess for the contour, and then it moved by the image driven forces to the boundaries of the desired region. There are two type of active contours models for segmentation edge based and region based. Edge based active contours use an edge detector, to find the boundaries of sub-regions and to attract the contours to detected boundaries. Region based active contours uses statistical information of image intensity within each subset instead of searching geometrical boundaries.

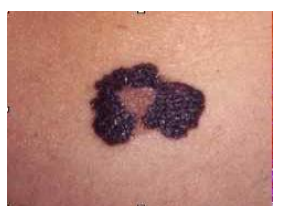

(a) Dermoscopic Image

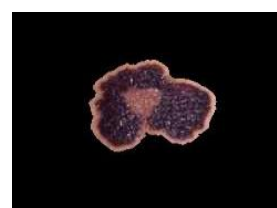

(b) Segmented Image
Fig.5 Segmentation 


\subsection{Feature Extraction}

Feature extraction is the method of extracting the unique feature of skin lesion. There are certain features like color, geometry, and size; which distinguish malignant melanoma from benign melanoma. Technique used for feature extraction is Gray Level Co-occurrence Matrix (GLCM) [1]. GLCM is useful tool for the image texture classification. It defined as a 2-D histogram of gray levels for a pair of pixels, which are separated by a fixed spatial relationship. Segmented image in gray scaled given as input. The features extracted based on GLCM are: Contrast, Correlation, Energy and Homogeneity. Three additional features Mean, Kurtosis and Skewness. Mean, Kurtosis and Skewness used for describes the geometry of the image.

For an image $P(i, j)$ where $I$ is number of rows and $j$ is number of Colum; with number of gray levels $G$, the features are expressed as

\subsubsection{Contrast}

It is the measure of local intensity variation of pixels.

$$
\text { Contrast }=\sum_{n=0}^{G-1} n^{2}\left\{\sum_{i=1}^{G} \sum_{j=1}^{G} P(i, j)\right\}
$$

\subsubsection{Correlation}

It is a measure of gray level linear dependency between the pixels at the specified positions relative to each other.

$$
C o=\sum_{i=0}^{G-1} \sum_{j=0}^{G-1} \frac{\{i \times j\} \times P(i, j)-\left\{\mu_{x} \times \mu_{y}\right\}}{\sigma_{x} \times \sigma_{y}}
$$

\subsubsection{Homogeneity}

Angular Second Moment (ASM) is measure of Homogeneity; and it is a measure of uniformity of an image.

$$
\text { Homogeneity }=\sum_{i=0}^{G-1} \sum_{j=0}^{G-1}\{P(i, j)\}^{2}
$$

\subsubsection{Energy}

Energy is defined based on a normalized histogram of the image. It shows how the gray levels are distributed, when the number of gray levels is low then energy is high.

\subsubsection{Mean}

$$
\text { Energy }=\sum_{i, j}^{G-1}\{P(i, j)\}^{2}
$$

It is simple athematic mean define as sum of observation divided by number of observation.

$$
\text { Mean }=\frac{x_{1}+x_{2}+x_{3}+\cdots x_{N}}{N}
$$

\subsubsection{Skewness}

Skewness means lack of symmetry, it is measure of asymmetry. Data is symmetric if it looks same to left and right about centre point. Positive skewness indicates a data with as asymmetric tail extending towards more positive values; Negative skewness indicates a data with an asymmetric tail extending towards more negative values.

$$
\text { Skewness }=\frac{\text { Mean }- \text { Mode }}{\text { Stander Diviation }}
$$

\subsubsection{Kurtosis}

Kurtosis is measure of sharpness or flatness of data compared to the normal distribution. Positive kurtosis indicates a relatively peaked distribution; Negative kurtosis indicates a relatively flat distribution. Data sets with higher Kurtosis tend to have peak near the mean.

$$
\text { Kurtosis }=\frac{\text { Fourt h central Moment }}{(\text { Stander Diviation })^{4}}-3
$$

\subsection{Artificial Neural Network Classifier}

Neural network is able to deal with highly complex problem very easily because of nonlinear capability of its neurons. ANN is used for the classification of the malignant melanoma from the benign melanoma. Network training is done by the Back Propagation Network Algorithm (BPN). The structure of the neural network classifier is of three layer structure containing Input Layer, Hidden Layer and Output Layer shown in fig. 6 . The Input layer consist of the input neurons, the value of neurons are parameters which are extracted in the feature extraction step. Hidden layer and Output layer contain adjustable weights according to error output in the classification. In BPN, input signals flows in forward direction and the output of the network is compared with the desired output. If output matches to desired one then no error signal is generated. If their mismatch an error signal is generated; it propagated backwards and weights are adjusted based on the gradient of error curve, which points in the direction to the local minimum.

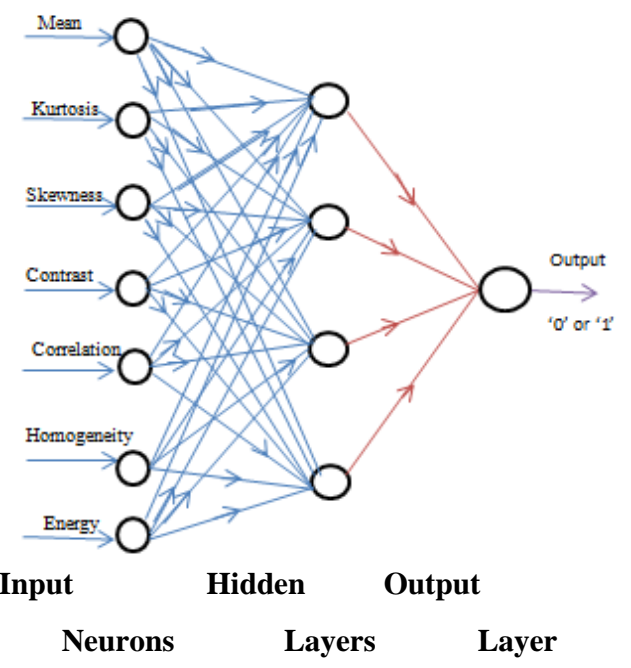

Fig.6 Artificial Neural Network Classifier

In BPN, the weights used at the beginning of the training are random in nature and its initial values are selected randomly. Training is done with the known values for the desired output, supervisory learning is used here. After training, network can perform decision making. When input signal passed through the forward network; according to the initial weight and activation function used, an output is generated. This output is compared with the desired output. If both do not match, than there is an error (8). The error is back-propagated and weights of hidden layer and output layer are adjusted. This process continues until error is zero or within set tolerable limits.

Error $=$ Desired Output - Actual Output 
In the proposed method, seven feature given as input to multilayer feed forward network with seven input neurons. Hidden layer with four hidden neurons; and Output with one output neurons. Activation function is Log sigmoid function, which gives output ' 0 ' for noncancerous condition and ' 1 ' for the cancerous condition. Neural network tool of MATLAB is used for the classification. The network is trained using known features data sets of malignant and benign melanoma images; and Training is repeated until the Mean Square Error (MSE) is at its minimum. Data sets of 50 Malignant Melanoma and benign melanoma images are tested; and output of the classifier is either ' 1 ' for cancerous condition or ' 0 ' for noncancerous condition.

\section{RESULTS}

Dermoscopic images of Malignant Melanoma and Benign Melanoma are collected from Internet and Clinics. These medical images processed further for hair removal by Dull Razor software, segmented by active contour approach. Feature extraction is done by MATLAB software. Calculated features are shown in table 2; it is given as input neurons of Artificial Neural Network, which gives output ' 1 ' or ' 0 '; ' 1 ' for cancerous or Malignant Melanoma and ' 0 ' for Benign Melanoma. Neural Network is simulated using in MATLAB software. For testing, 50 cases are considered. ANN classifies data of images as cancerous and noncancerous. 29 were classified as cancerous and 21 as noncancerous. The Confusion matrix of classification shown in fig.7.The accuracy of the proposed technique is $90 \%$.

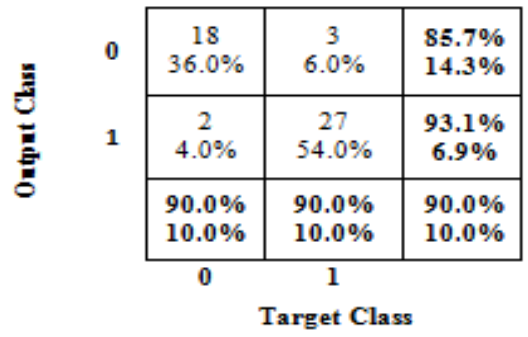

Fig.7 Confusion Matrix

\section{RESULT VALIDATION}

Validation of the result is done by Receiver Operating Characteristics (ROC) curve fig 8; it is commonly used for medical decision making. ROC is a technique used for systematized binary classifiers and their performance. Area under the Curve (AUC) shows the accuracy of the classifier [11].

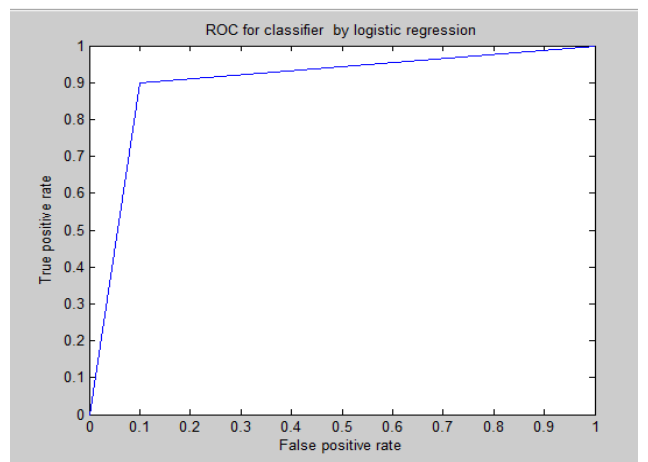

Fig 8 ROC curve
The results obtain by the proposed method were compared with Diagnosis report prepared by doctors. Among the 50 images used for testing, 30 were cancerous and 20 noncancerous according to doctors' report. This report is taken as the reference to validate the obtain result. The ANN classifier gives the output of 29 cancerous and 21 noncancerous cases. There were 5 misclassifications. The result shows that proposed technique has an accuracy of $90 \%$. The error can be reduced by increasing the training session with more number of images to ANN classifier.

\section{CONCLUSIONS AND FUTURE WORK}

Classification of Skin Melanoma is proposed and it is better diagnosis method. This method helps the patient to identify the skin cancer even without going to hospitals; and save both time and life of the patient. This diagnosis method includes both Image Processing and Artificial Neural Network with the accuracy of $90 \%$. By varying the technique and training algorithms like finding more parameters, changing image processing technique or by changing ANN training algorithm the accuracy can be improved.

\section{REFERENCES}

[1] J Abdul Jaleel, Sibi Salim, Aswin.R.B "Computer Aided Detection of Skin Cancer" International Conference on Circuits, Power and Computing Technologies, pp 1137-1142, 2013

[2] Ho Tak Lau and Adel AI-Jumaily, "Automatically Early Detection of Skin Cancer: Study Based on Neural Network Classification" , International Conference of Soft Computing and Pattern Recognition, IEEE, pp 375-380, 2009.

[3] Fikret Ercal, Anurag Chawla, William V. Stoecker, Hsi-Chieh Lee, and Randy H.Moss, "Neural Network Diagnosis of Malignant Melanoma From Color Images", IEEE Transactions on Biomedical Engineering. vol. 41, No. 9,1994

[4] National Cancer Institute, What You Need to Know Abour Dysplastic Nevi, NIH Publication 91-3133, Reprinted Oct. 1990.

[5] T. Tanaka, R. Yamada, M. Tanaka, K. Shimizu, M. Tanaka, "A Study on the Image Diagnosis of Melanoma" , IEEE Trans. on Image Processing, pp. 1010-1024, June 2004.

[6] M. Airouche, L. Bentabet, M. Zelmat, "Image Segmentation using Active Contour Model and Level set Method Applied to Detect Oil Spills" Proceedings of the World Congress on Engineering 2009 Vol I WCE 2009, July 1 - 3, 2009, London, U.K.

[7] T. F. Chan, L. A. Vese, "Active contours without edges". IEEE Transactions on Image Processing, Volume 10, Issue 2, pp. 266-277, 2001.

[8] V. Caselles, R. Kimmel, G. Sapiro, "Geodesic active contours". International Journal of Computer Vision, Volume 22, Issue 1, pp. 61-79, 1997.

[9] R. T. Whitaker, "A level-set approach to 3d reconstruction from range data". International 
Journal of Computer Vision, Volume 29, Issue 3, pp.203-231, 1998,

[10] British Columbia Cancer Agency, Tim Lee " Dull Razor for Windows", Copyright (c) 2003

[11] Kevin Woods, Kevin W. Bowyer "Generating ROC Curves for Artificial Neural Networks". IEEE transactions on medical imaging, volume 16, no. 3 , pp 329-337, June 1997.

[12] Bino Sebastian, A. Unnikrishnan and Kannan Balakrishnan, "Grey Level Co-occurrence Matrices: generalisation and some new features". International Journal of Computer Science, Engineering and
Information Technology (IJCSEIT), Vol.2, No.2, pp 151-157, April 2012.

[13] D. Baswaraj, Dr. A. Govardhan and Dr. P.Premchand ."Active Contours and Image Segmentation: The Current State of the age". Global Journal of Computer Science and Technology Graphics \& Vision, Volume 12 Issue 11 Version 1.0 Year 2012.

[14] American cancer society, "Cancer Facts and Figure 2015".

[15] World Health Organization "Cancer", 2015.

\section{APPENDIX}

Table 2 Results of Test Images

\begin{tabular}{|c|c|c|c|c|c|c|c|c|}
\hline Mean & Kurtosis & Skewness & Contrast & Correlation & Energy & $\begin{array}{c}\text { Homogen } \\
\text { eity }\end{array}$ & $\begin{array}{l}\text { Out } \\
\text { put }\end{array}$ & Cases \\
\hline 161.3019 & -3 & -3.8133 & 0.4888 & 0.9071 & 0.4853 & 0.911 & 1 & Cancerous \\
\hline 148.2178 & -3 & -1.3168 & 0.426 & 0.925 & 0.2935 & 0.8907 & 1 & Cancerous \\
\hline 162.2136 & -2.9959 & -12.6185 & 0.0167 & 0.9595 & 0.9741 & 0.9984 & 0 & Non-cancerous \\
\hline 135.1221 & -3 & -0.8622 & 0.2963 & 0.9399 & 0.3061 & 0.9075 & 1 & Cancerous \\
\hline 142.6768 & -3 & -2.0077 & 0.3458 & 0.9305 & 0.418 & 0.9258 & 0 & Non-cancerous \\
\hline 163.9342 & -2.9997 & -0.9832 & 0.1369 & 0.9046 & 0.8147 & 0.9728 & 1 & Cancerous \\
\hline 111.6023 & -3 & -1.3432 & 0.6281 & 0.8034 & 0.0988 & 0.7649 & 0 & Non-cancerous \\
\hline 138.8078 & -2.9999 & -1.9097 & 0.2108 & 0.9106 & 0.6949 & 0.9577 & 1 & Cancerous \\
\hline 124.6400 & -3 & -2.1246 & 0.415 & 0.903 & 0.3902 & 0.9125 & 0 & Non-cancerous \\
\hline 174.6732 & -2.9989 & -8.7248 & 0.0895 & 0.8785 & 0.9274 & 0.9898 & 1 & Cancerous \\
\hline 181.2678 & -2.9984 & -12.5772 & 0.0506 & 0.915 & 0.9632 & 0.9956 & 0 & Non-cancerous \\
\hline 147.3914 & -3 & -1.8978 & 0.2338 & 0.9574 & 0.3508 & 0.957 & 1 & Cancerous \\
\hline 146.9637 & -2.9921 & -13.9926 & 0.0135 & 0.9456 & 0.9757 & 0.9983 & 0 & Non-cancerous \\
\hline 107.1261 & -2.9999 & -1.4555 & 0.1661 & 0.9118 & 0.4033 & 0.9563 & 1 & Cancerous \\
\hline 173.421 & -2.9996 & -6.1311 & 0.0951 & 0.9128 & 0.8817 & 0.9875 & 0 & Non-cancerous \\
\hline 150.7939 & -2.9999 & -3.394 & 0.2726 & 0.8999 & 0.6516 & 0.9469 & 1 & Cancerous \\
\hline 160.3541 & -2.9995 & -6.7231 & 0.0768 & 0.9327 & 0.9082 & 0.9919 & 0 & Non-cancerous \\
\hline 130.1925 & -2.9999 & -0.9725 & 0.4343 & 0.8663 & 0.4042 & 0.9062 & 1 & Cancerous \\
\hline 158.5132 & -2.9999 & -1.7486 & 0.1872 & 0.9202 & 0.6999 & 0.9695 & 0 & Non-cancerous \\
\hline 165.0065 & -3 & -2.6875 & 0.1414 & 0.9629 & 0.598 & 0.9687 & 1 & Cancerous \\
\hline 135.9579 & -3 & -1.7375 & 0.1788 & 0.9614 & 0.3457 & 0.9477 & 0 & Non-cancerous \\
\hline 175.3509 & -2.9988 & -4.8184 & 0.0617 & 0.9084 & 0.9373 & 0.993 & 0 & Non-cancerous \\
\hline 156.0926 & -2.9999 & -4.0292 & 0.0963 & 0.9612 & 0.792 & 0.9793 & 1 & Cancerous \\
\hline 174.7291 & -3 & -3.5274 & 0.6469 & 0.8811 & 0.477 & 0.8909 & 1 & Cancerous \\
\hline 181.9671 & -2.9997 & -6.3255 & 0.0918 & 0.9305 & 0.9098 & 0.9921 & 0 & Non-cancerous \\
\hline 126.9674 & -2.9996 & -3.2274 & 0.0761 & 0.9228 & 0.7915 & 0.985 & 1 & Cancerous \\
\hline 150.7323 & -2.9999 & -3.7055 & 0.0519 & 0.9808 & 0.7652 & 0.9915 & 1 & Cancerous \\
\hline 190.0455 & -2.9998 & -5.978 & 0.102 & 0.9452 & 0.9067 & 0.9897 & 0 & Non-cancerous \\
\hline 142.9023 & -3 & 0.5255 & 0.3442 & 0.8959 & 0.5434 & 0.9237 & 1 & Cancerous \\
\hline 174.5183 & -3 & -1.9265 & 0.2148 & 0.9342 & 0.5368 & 0.9608 & 1 & Cancerous \\
\hline 142.9552 & -2.9988 & -2.4709 & 0.0423 & 0.936 & 0.8756 & 0.9937 & 1 & Cancerous \\
\hline 166.2316 & -3 & -2.3702 & 0.2815 & 0.9232 & 0.5413 & 0.9278 & 1 & Cancerous \\
\hline
\end{tabular}


International Journal of Computer Applications (0975 - 8887)

Volume 128 - No.10, October 2015

\begin{tabular}{|c|c|c|c|c|c|c|c|c|}
166.2793 & -2.9969 & -11.3022 & 0.0256 & 0.941 & 0.9676 & 0.9977 & 0 & Non-cancerous \\
\hline 155.0032 & -3 & -3.2401 & 0.2167 & 0.9397 & 0.5363 & 0.9561 & 1 & Cancerous \\
\hline 152.4697 & -3 & -1.0915 & 0.6903 & 0.893 & 0.2474 & 0.8563 & 1 & Cancerous \\
\hline 156.7985 & -2.9997 & -7.4773 & 0.1413 & 0.9028 & 0.9133 & 0.988 & 0 & Non-cancerous \\
\hline 201.1377 & -2.9999 & -4.4527 & 0.1498 & 0.9432 & 0.7944 & 0.9788 & 1 & Cancerous \\
\hline 192.9368 & -2.9997 & -6.8634 & 0.0934 & 0.9389 & 0.9177 & 0.9943 & 0 & Non-cancerous \\
\hline 173.0937 & -2.9992 & -7.3494 & 0.0435 & 0.9432 & 0.9031 & 0.9907 & 1 & Cancerous \\
\hline 152.311 & -3 & -2.2257 & 0.3268 & 0.9341 & 0.5169 & 0.9372 & 1 & Cancerous \\
\hline 166.5393 & -2.9996 & -5.7123 & 0.1412 & 0.8891 & 0.9091 & 0.987 & 0 & Non-cancerous \\
\hline 141.0982 & -2.9999 & -3.7539 & 0.0736 & 0.9673 & 0.7349 & 0.9888 & 1 & Cancerous \\
\hline 164.133 & -2.9992 & -4.1888 & 0.0498 & 0.9329 & 0.897 & 0.9903 & 1 & Cancerous \\
\hline 186.6169 & -2.9982 & -13.0841 & 0.0455 & 0.925 & 0.9754 & 0.9978 & 0 & Non-cancerous \\
\hline 135.1551 & -2.9998 & -0.8472 & 0.1834 & 0.8787 & 0.7065 & 0.9574 & 1 & Cancerous \\
\hline 148.367 & -2.9997 & -5.7838 & 0.1137 & 0.919 & 0.901 & 0.9888 & 0 & Non-cancerous \\
\hline 135.663 & -2.9999 & -4.0776 & 0.128 & 0.9455 & 0.7797 & 0.9806 & 1 & Cancerous \\
\hline 164.1204 & -3 & -2.0126 & 0.6591 & 0.8881 & 0.4161 & 0.8806 & 0 & Non-cancerous \\
\hline 199.1242 & -2.9999 & -1.0889 & 0.2168 & 0.9108 & 0.7752 & 0.9679 & 1 & Cancerous \\
\hline 166.4714 & -2.9923 & -9.1722 & 0.0221 & 0.9051 & 0.9732 & 0.9976 & 0 & Non-cancerous \\
\hline
\end{tabular}

